\title{
Impact of obesity and gender differences on electrodermal activities
}

\author{
Haval Y. Y. Aldosky \\ Department of Physics, College of Science, University of Duhok, Duhok, Iraq
}

\begin{abstract}
The widespread use of electrodermal activity (EDA) as a measuring tool in sympathetic nervous system activity researches has resulted in a wide variety of publications on EDA. Yet, the influences of obesity and gender differences in the responses and levels of EDA have not been studied carefully. This study aims to investigate the impacts of obesity and gender differences on EDA parameters. To this end, EDA responses and levels were recorded from 36 (18 males and 18 females) apparently healthy adult subjects nearly half of them are obese. EDA measures were induced by three different stimuli and the EDA responses and levels were compared as a function of obesity and gender differences for all stimuli. It was found that EDA parameters, in particular, skin conductance responses (SCRs) were significantly different between obese and non-obese subjects for both genders. In addition, it was found that the change in skin susceptance levels (SSLs) was statistically significant only among females. The investigation also showed that the gender difference has a crucial role in skin potential levels (SPLs) for obese subjects. The outcome results led to the conclusion that the phasic SCRs were strongly related to BMI, while tonic SPLs were related to gender differences.
\end{abstract}

Key words: Obesity - Gender differences - Phasic EDA — Tonic EDA — Skin conductance response

\begin{abstract}
Abbreviations: BMI, body mass index; ECG, electrocardiogram; EDA, electrodermal activity; SC, skin conductance; SCLs, skin conductance levels; SCRs, skin conductance responses; SP, skin potential; SPLs, skin potential levels; SPRET, skin potential relative early turn; SPRs, skin potential responses; SS, skin susceptance; SSLs, skin susceptance levels; SSRs, skin susceptance responses.
\end{abstract}

\section{Introduction}

Electrodermal activity (EDA) has become a valuable tool in behavioral medicine as a biomarker for individual characteristics of emotional responsiveness. It represents an important index for direct examination of axis of stress-related effects on bodily function, and as a potential pathway for treatment of psychosomatic through biofeedback training (Critchley and Nagai 2013). The progress in recording and analyzing EDA measurement data has recently increased the interest for possible applications in various clinical settings such as operation room, recovery and intensive care unit where the monitoring of the autonomous nervous system activity becomes vital (Theodoros 2014). The most frequently used and studied parameter of EDA is skin conductance (SC)

Correspondence to: Haval Y. Yacoob Aldosky, Department of Physics, College of Science, University of Duhok, Duhok, Iraq E-mail: yacoobaldosky@uod.ac which has been used for physiological and psychophysiological measurements due to a well-known link with the sympathetic nervous system (Boucsein 2012; Tonacci et al. 2019). Regarding the evaluation of the autonomic nervous system, measurements of skin potential (SP) are often preferred (Kucera et al. 2004; Zygmunt and Stanczyk 2009). Furthermore, the sympathetic nervous system is associated not only with the skin conductance but also with the skin susceptance (i.e., imaginary part of the skin impedance) (Greco et al. 2016). The low-frequency susceptance method has proven to be the most appropriate measure of the degree of skin hydration (Martinsen and Grimnes 2008).

However, there are several important factors that influence EDA which should be considered during the process of monitoring. These factors are categorized into two types, participant's characteristics and environmental conditions. It is known that obesity causes many changes in skin physiology and the gender differences results in various levels of sweat gland activation (Yosipovitch et al. 2007; Gagnon 
and Kenny2012). Therefore, gender differences and obesity are chosen as participant characteristics since they showed a remarkable impact on the EDA. In particular, this impact and its mechanism have not yet been studied comprehensively. According to literatures, a confusion point exist on how gender differences and obesity affect EDA. The environmental conditions include temperature (Sano et al. 2014) and humidity (Bari et al. 2018).

There has been much controversy over the effect of gender differences on EDA responses and levels. For example, Isen et al. (2010) found that the skin conductance responses (SCRs) revealed unexpected high sensitivity to stimuli in males over females. Similarly, Oliver et al. (2017) found that gender differences have an important role recording SCR as the values of SCRs of females were significantly less than the males post stimuli. In contrast, Chentsova and Tsai (2007) found that female participants showed greater increases in skin conductance levels (SCLs) than their males counterparts, suggesting that they are more physiologically aroused than males. Similarly, Gaviria et al. (1969) found that females showed variances in skin potential levels (SPLs) significantly higher than the variances that are shown by males. Whereas, the capacitive properties of skin and sweat glands were given less attention by researchers comparing to SC and SP for both gender differences and obesity. With regard to obesity factor, few studies have focused on association between obesity and EDA responses. According to this study, overweight and obesity can be defined as abnormal or excessive fat accumulation that represents a great risk to health. A crude population measure of obesity is the BMI, a person's weight (in kilograms) divided by the square of his or her height (in meters). A person with a BMI of $30 \mathrm{~kg} / \mathrm{m}^{2}$ or more is generally considered obese (WHO2019). Park et al. (2015) demonstrated that the skin conductance levels (SCLs) were not correlated with obesity for levels of stress. Likewise, Oliver et al. (2017) demonstrated that the skin conductance responses (SCRs) reactivity to stress were not affected by obesity. There appears that only few and limited studies have been conducted to investigate the impacts of gender differences and obesity on EDA responses and levels. These studies suffer from two main limitations. The first limitation is, the avaiable studies focused on skin conductance (Park et al. 2015) rather than all EDA parameters. The second limitation is, almost all of the conducted studies implemented on obese females only. In this study, comprehensive investigations on the impacts of gender differences and obesity are done on EDA responses and levels with three stimuli. The objective is to achieve two major goals. The first is to exam whether EDA is significantly influenced by gender differences and/ or obesity. The second is to investigate which EDA phasic or tonic is more likely to be evoked by these two factors (gender differences and obesity).

\section{Materials and Method}

Subjects

A total of 36 students have recruited as test subjects from the University of Duhok with age 19-24 years (mean age, 20.22 \pm 1.98 years). The students were divided into two groups, eighteen students for each according to their gender. Each group was intentionally assigned into two subgroups for males and females. One subgroup contains 9 non-obese (BMI 18.5-24.9 $\mathrm{kg} / \mathrm{m}^{2}$ ) and the other subgroup contains 9 obese subjects $\left(\mathrm{BMI}>30 \mathrm{~kg} / \mathrm{m}^{2}\right)$ based on values provided by the WHO.

\section{Measurements}

Electrodermal responses and levels were recorded simultaneously at the same electrodes (Kendall Kittycat 1050NPSM $\mathrm{Ag} / \mathrm{AgCl}$ solid gel ECG) on the same skin sites by employing a computerized system. The computerized system was made up of a small front-end electronic box connected to a PC laptop by means of a National Instruments DAQ cardNI USB-6211 and software was developed and executed in programing environment Lab VIEW, v. 14 as in the system presented in Bari et al. (2018).

Test subjects sat on a comfortable chair throughout the experiment and special care was given to maintain the room temperature $\left(22-23^{\circ} \mathrm{C}\right)$ as recommended (Boucsein 2012). Five minutes were needed to stabilize the electrodes before starting the process of recording. The responses and levels of the skin conductance (SC), skin potential (SP) and skin susceptance (SS) as functions of three different external stimuli that cause mental stress were recorded for the obese and normal (non-obese) groups. The stimuli were as follows:

a) Cognitive (mathematical calculations), for instance, asking the test subject a question such what is the result of subtracting 17 from 100, and the answer should be provided in $5 \mathrm{~s}$,

b) The vision (fright) test subject was asked to look at a scary photo for $3 \mathrm{~s}$,

c) Deep breath, the test subject was asked to take a deep breath for $4 \mathrm{~s}$.

Before and after each of the three stimuli, there was a relaxation time of $60 \mathrm{~s}$ in order to obtain the baseline of the EDA measurements; thus yielding a total of $492 \mathrm{~s}$ for recording each subject. At the relaxation time, subjects were asked to relax, to remain awake and to avoid bodily movement. Talking was not allowed for the subjects during the whole session of data collection.

\section{Ethical approval}

The protocol complied with all the relevant national regulations, institutional policies and in accordance with the 
Table 1. Descriptive statistics of the physical characteristics for the subjects in terms of BMI and gender differences

\begin{tabular}{lccccc}
\hline Characteristic & $\begin{array}{c}\text { Non-obese } \\
(n=18)\end{array}$ & $\begin{array}{c}\text { Obese } \\
(\boldsymbol{n}=18)\end{array}$ & $\begin{array}{c}\text { Male } \\
(n=18)\end{array}$ & $\begin{array}{c}\text { Female } \\
(n=18)\end{array}$ & Significant differences \\
\hline Age (years) & $19.55 \pm 1.54$ & $21.06 \pm 2.21$ & $20.38 \pm 2.11$ & $20.05 \pm 1.89$ & NS \\
Body weight $(\mathrm{kg})$ & $60.81 \pm 11.58$ & $103.39 \pm 28.94$ & $86.69 \pm 34.18$ & $72.78 \pm 23.79$ & Obesity $^{*}$ (by design) \\
Height $(\mathrm{cm})$ & $167.75 \pm 9.71$ & $169.06 \pm 7.58$ & $173.88 \pm 6.71$ & $162.77 \pm 6.8$ & Gender $^{*}$ \\
BMI $\left(\mathrm{kg} / \mathrm{m}^{2}\right)$ & $21.57 \pm 3.31$ & $36.20 \pm 8.47$ & $28.67 \pm 11.1$ & $27.5 \pm 8.01$ & Obesity $^{*}$ (by design) \\
\hline
\end{tabular}

NS, non significant; ${ }^{*}$ significant; BMI, body mass index.

Helsinki Declaration and was approved by the Deanery of the College of Science at the University of Duhok. Informed consent has been obtained from each individual included in this study.

\section{Statistical analysis}

The Statistical Package for Social Sciences (SPSS) was used for data analysis. Data were presented as mean values and standard deviations for the physical characteristics of subjects. The differences in the EDA responses and levels were evaluated statistically in terms of gender differences and BMI by using Mann-Whitney U Test results. The level of statistical significance was set as $p$-value $<0.05$ (Exact 2-tailed).

Table 2. Electrodermal responses and levels obtained by calculating the amplitude and the average of onsets of the three responses for all participants

\begin{tabular}{|c|c|c|c|}
\hline $\begin{array}{l}\text { EDA } \\
\text { parameter }\end{array}$ & $n$ & Comparison & $p$ \\
\hline \multirow{3}{*}{$\begin{array}{l}\text { SCR } \\
(\mu \mathrm{s})\end{array}$} & 36 & Obese $v s$. non-obese & 0.017 \\
\hline & 18 & Male vs. female (non-obese) & NS \\
\hline & 18 & Male vs. female (obese) & NS \\
\hline \multirow{3}{*}{$\begin{array}{l}\text { SPR } \\
(\mathrm{mV})\end{array}$} & 36 & Obese $v s$. non-obese & NS \\
\hline & 18 & Male $v s$. female (non-obese) & NS \\
\hline & 18 & Male vs. female (obese) & NS \\
\hline \multirow{3}{*}{$\begin{array}{l}\text { SSR } \\
(\mu \mathrm{s})\end{array}$} & 36 & Obese $v s$. non-obese & NS \\
\hline & 18 & Male vs. female (non-obese) & NS \\
\hline & 18 & Male vs. female (obese) & NS \\
\hline \multirow{3}{*}{$\begin{array}{l}\text { SCL } \\
(\mu \mathrm{s})\end{array}$} & 36 & Obese $v s$. non-obese & NS \\
\hline & 18 & Male $v s$. female (non-obese) & NS \\
\hline & 18 & Male vs. female (obese) & NS \\
\hline \multirow{3}{*}{$\begin{array}{l}\text { SPL } \\
(\mathrm{mV})\end{array}$} & 36 & Obese $v s$. non-obese & NS \\
\hline & 18 & Male vs. female (non-obese) & NS \\
\hline & 18 & Male $v s$. female (obese) & 0.031 \\
\hline \multirow{3}{*}{$\begin{array}{l}\text { SSL } \\
(\mu \mathrm{s})\end{array}$} & 18 & Obese $v s$. non-obese (in female) & 0.030 \\
\hline & 18 & Male vs. female (non-obese) & NS \\
\hline & 18 & Male vs. female (obese) & NS \\
\hline
\end{tabular}

Mann-Whitney U Test, $p$ value/Exact (2-tailed). NS, non significant.

\section{Results}

\section{Physical characteristics of the subjects}

In order to show physical characteristics of the subjects, descriptive statistics (means and standard deviations) and $p$ values for the subjects in terms of BMI were determined as shown in Table 1. According to this table, there was no significant difference in age and height between the obese and non-obese groups. The mean BMI of the non-obese subjects was $21.57 \pm 3.31 \mathrm{~kg} / \mathrm{m}^{2}$ and for the obese was 36.20 $\pm 8.47 \mathrm{~kg} / \mathrm{m}^{2}(p<0.05)$. In terms of gender differences, the table shows that there was also no significant difference in age between males and females, although males were taller than females but this has no remarkable effect of height on obesity as shown in Table 1 .

\section{Amplitudes of EDA responses}

A significant difference in SCRs amplitude between the groups was found. Figure 1 shows the median value of SCRs amplitude response to the stimuli with respect to the BMI. SCRs amplitudes were significantly higher in obese subjects as compared to non-obese subjects as shown in Table 2. Skin

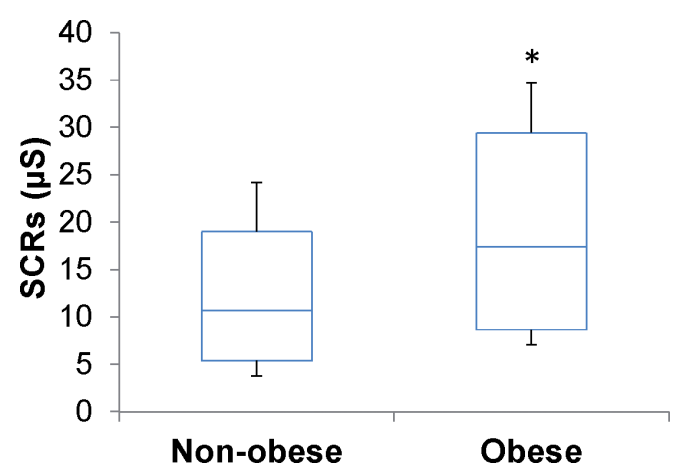

Figure 1. Skin conductance response (SCR) in the non-obese and obese subjects. Data are presented as box and whisker plots with the $75^{\text {th }}$ percentile (upper box), median (inner line), $25^{\text {th }}$ percentile (lower box) and minimum and maximum values (tails). $n=36$; ${ }^{\star} p<0.05$ vs. non-obese subjects. 


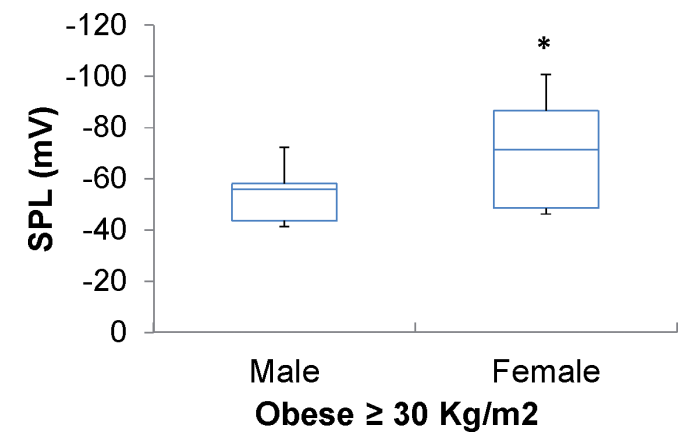

Figure 2. Skin potential level (SPL) in the obese subjects (male and female). Data are presented as box and whisker plots with the $75^{\text {th }}$ percentile (upper side), median (inner line), $25^{\text {th }}$ percentile (lower side) and minimum and maximum values (tails). $n=18$; ${ }^{*} p<0.05 v s$. male subjects.

potential responses (SPRs) and skin susceptance responses (SSRs) amplitudes changed as results of all the three stimuli. However, these changes were neither statistically significant with BMI nor with gender differences as indicated by MannWhitney $\mathrm{U}$ test. This means that the amplitudes of SCRs are influenced only by BMI.

\section{EDA levels}

The tonic components (levels) of EDA were also assessed. There was no significant difference in SCLs neither with regard to BMI nor to gender differences. A significant difference in SPLs between males and females of the obese group was found as shown in Figure 2, while no difference was found between males and females in non-obese group. For SSLs, significant differences were observed for BMI in females only $(p<0.05)$ as indicated in Figure 3. However,

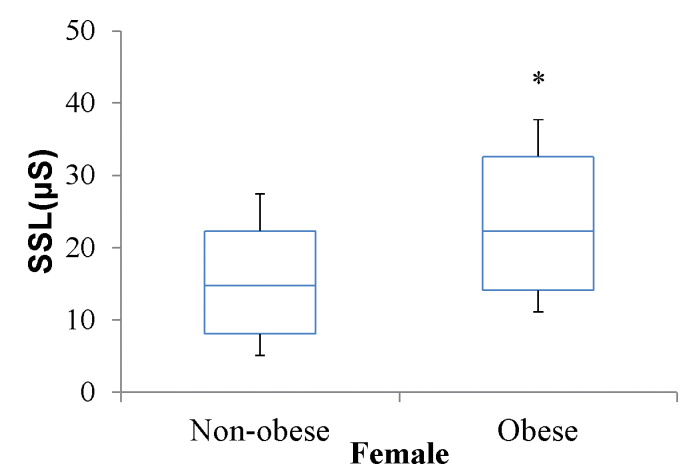

Figure 3. Skin susceptance level (SSL) in the non-obese and obese subjects. Data are presented by box and whisker plots with $75^{\text {th }}$ percentile (upper side), median (inner line), $25^{\text {th }}$ percentile (lower side) and minimum and maximum values (tails). $n=18 ;{ }^{*} p<0.05$ $v s$. non-obese subjects.

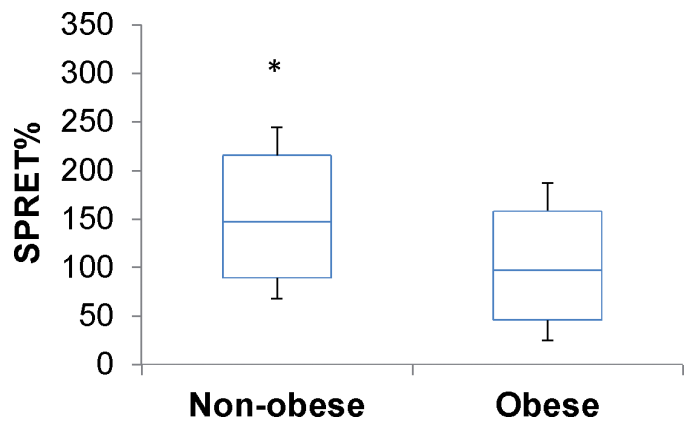

Figure 4. SPRET percentage in the non-obese and obese subjects. Data are presented by box and whisker plots with $75^{\text {th }}$ percentile (upper side), median (inner line), $25^{\text {th }}$ percentile (lower side) and minimum and maximum values (tails). $n=36$; ${ }^{*} p<0.05 v s$. obese subjects.

no significant differences in SSLs were observed with regard to gender differences.

\section{Skin potential relative early turn (SPRET)}

SPRET, which is the relative time difference between the turning points or peaks of the SPR and SCR waveforms was calculated and statistically analyzed. It was found that SPRET is influenced by BMI. Figure 4 shows the percentage value of SPRET for all SCR and SPR responses from all test subjects and it also shows that SPRET values for non-obese subjects were higher that these for obese subjects. This is indicated by the Mann-Whitney $U$ test analysis which yields a significant $(p<0.05)$ difference between the non-obese and obese groups.

\section{Discussion}

Generally, the results demonstrate that both obesity and gender differences influence the EDA responses and levels in one way or another. The interesting result is that all stimuli were able to evoke SCRs amplitude in all test subjects which means that the sympathetic nervous system was activated by the three stimuli. The increase in SCRs amplitudes was significantly larger for obese compared to the non-obese group. This result is in full agreement with that reported by (Spraul et al. 1993). However, Kronholm et al. (1993) reported another controversial result. To the author view, the results of this study is more realistic because the increase in the amplitude of SCRs can be justified by the fact that the obese subjects have larger skin folds and sweat more profusely because of the thick layers of subcutaneous fat (Yosipovitch et al. 2007; Shipman and Millington 2011). According to all models of EDA, the 
SCRs amplitudes are based completely on the sweat glands through the epidermis, which alters their conductance according to the degree to which they are filled with sweat (Boucsein 2012).

Also, the results demonstrate that the SPLs levels were significantly affected by gender differences in obese subjects. The SPL level is negatively increased (more negative) in obese females more compared to males when sweat glands are activated by stimuli. A possible explanation for this is that in many cases female subjects display a higher tonic EDA, while male subjects tend to show a greater electrodermal reactivity under conditions of stimulation (Boucsein 2012). Also, tonic SSL was higher in obese females under all stimuli compared to non-obese. The capacitive elements certainly play an important role in tonic EDA. Whereas, the phasic changes in capacitive properties of skin, have a minor role (Boucsein 2012). The increase in SSLs is indicator of increasing the electrical capacitance of the skin, which is proportional to the moisture content or hydration of the corneum for normal individuals (Martinsen et al. 2008). The elevation in skin capacitance with high BMI in females could be attributed to the contribution of the capacitance of the deeper tissues that cause an increase in the total capacitance and to the thickness of keratin layer, the thinner the keratin the higher the capacitance of the skin (Lawler et al. 1960). In addition, it was found that SPRET values were modified with respect to obesity. In both obese and non-obese subjects, no negative SPRET was obtained. SPRET for non-obese subjects tends to be close to $100 \%$, when the SPR is positive bi-phasic and comes from a duct that is already filled to its limp capacity before secretion. During sweating (while ducts are filled), the SPR peak occurs earlier than the SCR peak when the hydraulic capacity is low, so SPRET is higher and this is in accordance with an earlier study by Tronstad et al. (2013). While, SPRET for obese subjects tend to be close to $0 \%$, (in case of negative mono-phasic SPR) when the SPR is very similar to the SCR and comes from an empty duct that is filled without increasing intraductal pressure.

In general, the results of SPRs, SSRs and SCLs parameters were not significantly affected by obesity or by gender difference. This could be due to the small sample size, and/ or low power in detecting small differences (i.e. lower than a few microsiemens) between the groups. These results don't come in agreement with those which have been reported by Neufeld and Davidson (1974) and Boucsein (2012) who claimed that there was a significant change in SCLs and SPLs with respect to gender differences. This disagreement between the results of this study and the previous studies may be due to the change in the environmental conditions such as temperature, relative humidity as well as age of subjects. In this study, these factors were kept under control.

\section{Conclusion}

The potential impact of obesity and gender based differences on EDA responses and levels in apparently healthy university students was investigated. The outcome results indicate that SCRs are closely related to obesity. Given the fact that SCRs are an important psychophysiological index for evaluating human behavior, obesity should be considered in phasic SCR measurements whereas the obese subject possesses reduced sympathetic and parasympathetic nerve activities. which means that obesity should be taken into account in phasic SCRs measurements. Concerning the skin potential, tonic SPLs were found to be significantly high in female subjects compared to males while measures of phasic SPRs activation were not. Tonic SSL has also found to be significantly high in only obese female subjects compared to non-obese females. It can be concluded that gender based differences are likely to affect the tonic rather than phasic EDA. These findings need to be confirmed using a larger sample size. In addition, more systematic investigations are needed on the role of capacitive elements that have an important role in the generation of an EDA in terms of BMI and gender differences.

\section{Limitations}

The main strength of this study is the simultaneous recording of the measurement for various EDA parameters on human skin and concurrently at the same skin site. However, some limitations should also be considered. First, BMI was used as a parameter for measuring obesity. However, this is the most useful epidemiological and clinical parameter used to define obesity in most studies. Second, the relatively small sample size, which does not allow for strong conclusions, but gives important indicators that can guide future work.

Conflict of interest. The author has declared no conflict of interest.

Acknowledgments. The author would like to thank deeply all the staff members at Physics Department, College of Science, University of Duhok for their assistance in data collection. Also thanks are due to the students who have shown their willingness to be the test subjects of this study. Last but not least, great thanks are due to Professor Ørjan Grøttem Martinsen (University of Oslo, Norway) for providing the necessary equipment to conduct this study.

\section{References}

Bari DS, Aldosky HYY, Tronstad C, Kalvøy H, Martinsen ØG (2018): Electrodermal responses to discrete stimuli measured by skin conductance, skin potential, and skin susceptance. Skin Res. Technol. 24, 108-116 https://doi.org/10.1111/srt.12397 
Bari DS, Aldosky HYY, Tronstad C, Kalvøy H, Martinsen ØG (2018): Influence of relative humidity on electrodermal levels and responses. Skin Pharmacol. Physiol. 31, 298-307 https://doi.org/10.1159/000492275

Boucsein W. (2012): Electrodermal activity (2nd ed.). New York, NY, US: Springer Science Business Media https://doi.org/10.1007/978-1-4614-1126-0

Chentsova-Dutton Y E, Tsai J L. (2007): Gender differences in emotional response among European Americans and Hmong Americans. Cogn. Emot. 21, 162-181 https://doi.org/10.1080/02699930600911333

Critchley H., Nagai Y. (2013): Electrodermal Activity (EDA). In: Encyclopedia of Behavioral Medicine. Springer (Eds. Gellman MD, Turner JR), p. 9-13, New York, NY https://doi.org/10.1007/978-1-4419-1005

Gagnon D, Kenny GP (2012): Sex differences in thermoeffector responses during exercise at fixed requirements for heat loss. J. Appl. Physiol. 113, 746-757 https://doi.org/10.1152/japplphysiol.00637.2012

Gaviria B, Coyne L, Thetford PE (1969): Correlation of skin potential and skin resistance measures. Psychophysiology 5, 465-477 https://doi.org/10.1111/j.1469-8986.1969.tb02850.x

Greco A, Lanata A, Citi L, Vanello N, Valenza G, Scilingo EP. (2016): Skin admittance measurement for emotion recognition: A study over frequency sweep. Electronics 5, 46 https://doi.org/10.3390/electronics5030046

Isen J, Raine A, Baker L, Dawson M, Bezdjian S, Lozano DI (2010): Sex-specific association between psychopathic traits and electrodermal reactivity in children. J. Abnorm. Psychol. 119, 216-225 https://doi.org/10.1037/a0017777

Kronholm E, Alanen E, Hyyppä MT.(1993): Nocturnal Motor Activity in a Community Sample. Sleep 16, 565-571 https://doi.org/10.1093/sleep/16.6.565

Kucera P, Goldenberg Z, Kurca E. (2004): Sympathetic skin response: review of the method and its clinical use. Bratisl. Lek. Listy 105, 108-116

Lawler Jc., Davis Mj, Griffith Ec. (1960): Electrical characteristics of the skin. The impedance of the surface sheath and deep tissues. J. Invest. Dermatol. 34, 301-308 https://doi.org/10.1038/jid.1960.52

Martinsen ØG, Grimnes S, Nilsen JK, Tronstad C, Jang W, Kim H, Shin K, Naderi M, Thielmann F. (2008): Gravimetric method for in vitro calibration of skin hydration measurements. IEEE Trans. Biomed. Eng. 55, 728-732 https://doi.org/10.1109/TBME.2007.912651

Martinsen $\varnothing \mathrm{G}$ and Grimnes S. (2008). Long-term effect of some skin moisturizers. The Open Dermatology Journal 2, 87-89 https://doi.org/10.2174/1874372200802010087

Neufeld RW, Davidson PO. (1974): Sex differences in stress response: a multivariate analysis. J. Abnorm. Psychol.83, 178-85 https://doi.org/10.1037/h0036391

Oliver MD, Datta S, Baldwin DR (2017): A sympathetic nervous system evaluation of obesity stigma. PloS One 12, e0185703. https://doi.org/10.1371/journal.pone.0185703

Park AE, Huynh P, Schell, AM, Baker LA. (2015): Relationship between obesity, negative affect and basal heart rate in predicting heart rate reactivity to psychological stress between adolescents. Int. J. Psychophysiol. 97, 139-144 https://doi.org/10.1016/j.ijpsycho.2015.05.016

Sano A, Picard RW, Stickgold R. (2014): Quantitative analysis of wrist electrodermal activity during sleep. Int J. Psychophysiol. 94, 382-389 https://doi.org/10.1016/j.ijpsycho.2014.09.011

Shipman, AR, Millington GWM. (2011): Obesity and the skin. Br. J. Dermatol. 165, 743-750 https://doi.org/10.1111/j.1365-2133.2011.10393.x

Spraul M, Ravussin E, Fontvieille AM, Rising R, Larson DE, Anderson EA. (1993): Reduced sympathetic nervous activity. A potential mechanism predisposing to body weight gain. J. Clin. Invest. 92, 1730-1735 https://doi.org/10.1172/JCI116760

Theodoros A. (2014): Electrodermal activity: applications in perioperative care. IJMRHS 3, 687-695 https://doi.org/10.5958/2319-5886.2014.00418.4

Tonacci A, Billeci L, Calderoni S, Levantini V, Masi G, Milone A, Pisanoe S, Muratori P. (2019): Sympathetic arousal in children with oppositional defiant disorder and its relation to emotional dysregulation. J. Affect. Disord. 257, 207-213 https://doi.org/10.1016/j.jad.2019.07.046

Tronstad C, Kalvøy K, Grimnes S, Martinsen ØG. (2013): Waveform difference between skin conductance and skin potential responses in relation to electrical and evaporative properties of skin. Psychophysiology 50, 1070-1078 https://doi.org/10.1111/psyp.12092

Yosipovitch G, DeVore A, Dawn A, (2007): Obesity and the skin: skin physiology and skin manifestations of obesity. J. Am. Acad. Dermatol. 56, 901-916

https://doi.org/10.1016/j.jaad.2006.12.004

Zygmunt A, Stanczyk J (2009). Methods of evaluation of autonomic nervous system function. Arch. Med. Sci. 6, 11-18 https://doi.org/10.5114/aoms.2010.13500

Received: August 11, 2019

Final version accepted: September 4, 2019 